\title{
Extension of a Kinetic-Theory Approach for Computing Chemical-Reaction Rates to Reactions with Charged Particles
}

\author{
Derek S. Liechty* and Mark J. Lewis ${ }^{\dagger}$ \\ *NASA Langley Research Center, Aerothermodynamics Branch, Mail Stop 408A, Hampton, VA 23681 \\ University of Maryland, Department of Aerospace Engineering, Room 3179 Martin Hall, College Park, MD 20742
}

\begin{abstract}
Recently introduced molecular-level chemistry models that predict equilibrium and nonequilibrium reaction rates using only kinetic theory and fundamental molecular properties (i.e., no macroscopic reaction rate information) are extended to include reactions involving charged particles and electronic energy levels. The proposed extensions include ionization reactions, exothermic associative ionization reactions, endothermic and exothermic charge exchange reactions, and other exchange reactions involving ionized species. The extensions are shown to agree favorably with the measured Arrhenius rates for near-equilibrium conditions.
\end{abstract}

Keywords. DSMC, chemical reactions, ionization

PACS: 47.11.Mn, 47.40.-x, 51.10+y, 52.20.Hv, 82.20.Pm, 82.30.Cf

\section{INTRODUCTION}

As we consider missions, both manned and unmanned, where the (re)entry velocities result in shock-layer temperatures on the order of tens-of-thousands of degrees Kelvin, the importance of electronic energy levels and reactions involving charged species becomes more pronounced. Although the treatment of these processes using the direct simulation Monte Carlo (DSMC) method is not a new development[1-3], most of these methods are based on measured equilibrium rates which are always questionable at high temperature, especially when applied to nonequilibrium conditions. The primary reason for the inadequate state of chemical reaction modeling is the difficulty in accurately measuring the internal energy state specific reaction rates to validate theoretical models in the temperature range of interest. Therefore, most reaction rates are based on low-temperature equilibrium measurements and are fit to the most reliable measured data sets. In some cases, the uncertainty associated with the spread of these measurements exceeds one order of magnitude[4].

Molecular-Level chemistry models that predict equilibrium and non-equilibrium reaction rates using only kinetic theory and fundamental molecular properties (i.e., no macroscopic reaction-rate information) have recently been proposed[5-7] (in addition, a new vibrational relaxation model has been introduced that only relies on fundamental molecular properties and a single measure of vibrational collision number at a reference temperature as well as a new electronic energy relaxation model [8] following the kinetic-theory approach). Preliminary evaluations of the new chemistry model indicated that the resultant reaction rates are in very good agreement with measured Arrhenius rates for near-equilibrium conditions and with both measured rates and other theoretical models for far-fromequilibrium conditions[6]. The new chemistry model is extended in this paper to include various reactions involving charged species.

\section{DESCRIPTION OF NEW CHEMISTRY MODELS}

Bird[5], supported by Gallis[6], have proposed a set of molecular-level chemistry models based solely on fundamental properties of the two colliding molecules: their total collision energy, their quantized vibrational energy levels, and their molecular dissociation energies. These models link chemical-reaction cross-sections to the energyexchange process and the probability of transition between vibrational energy states. The Larsen-Borgnakke procedures and the principle of microscopic reversibility are then used to derive simple models for reactions. These models do not require any macroscopic data and they function by seeking to balance the fluxes into and out of each state, 
thus satisfying microscopic reversibility. As mentioned in the introduction, preliminary evaluations[6] indicated that the resultant reaction rates are in very good agreement with measured Arrhenius rates for near-equilibrium conditions and with both measured rates and other theoretical models for far-from-equilibrium conditions. Since reactions involving only neutral species have been covered in References [5-7], these will not be discussed herein. A new methodology for accurately reproducing exothermic reactions has only recently been introduced by Bird[7], but the previous detailed balance procedure for exothermic reactions [5] appears to be sufficient if the molecules have similar masses and the activation energy for the endothermic reaction is high enough (e.g. for real air). So, most reactions involving only atoms, molecules, and ions in air can be treated using the detailed balance procedure, but the reactions involving electrons will need to be revisited using the new methodology.

\section{Ionization}

The procedure for ionization is quite similar to that for dissociation. The difference is that ionization is linked to the electronic energy levels instead of vibrational energy levels as dissociation is. For ionization, the collision energy is defined as

$$
E_{c}=E_{\text {trans,pair }}+E_{\text {elec,particle }}
$$

where the first term on the right is the relative translational energy of the colliding pair and the second term is the electronic energy of the particle being considered for ionization. If the collision energy is greater than the ionization energy of the particle, ionization occurs.

\section{Associative Ionization}

Associative ionization reactions require more care. These reactions can be thought of occurring as

$$
A+B \rightarrow A B^{*} \rightarrow A B^{+}+e^{-}
$$

where $A B^{*}$ is an intermediate, excited molecule that may lead to the ionized species $A B^{+}$plus an electron. The first step is similar to the recombination procedure described in Ref. [6]. If the $A B^{*}$ molecule is in the ground vibrational state after a Larsen-Borgnakke trial redistribution of the collision energy, the intermediate molecule is "formed." In the second step, the dissociation energy is first added to the original collision energy. The new collision energy is then distributed among the internal energy modes of the $A B^{*}$ molecule and then this molecule is tested for ionization as described above. If the remaining collision energy after redistribution plus the electronic energy of the molecule is greater than the ionization energy of the molecule, the associative ionization reaction occurs. Since the reverse (exothermic) reaction occurs with an electron, the the new methodology of [7] should be used in place of the old detailed balance model of [5].

\section{Endothermic and Exothermic Charge Exchange}

Charge exchange reactions are similar to the exchange reactions discussed in Ref. [6]. The endothermic reaction

$$
A+B^{+} \rightarrow A^{+}+B
$$

takes place when the electronic energy level of the colliding neutral particle, $A$, after a trial Larsen-Borgnakke redistribution of the sum of the relative translational energy and electronic energy of the neutral particle is equal to the electronic energy level with energy just above the activation energy of the reaction.

Since the exothermic charge exchange reaction occurs with atoms/molecules of similar mass, the detailed balance model[5] is acceptable for use, but should be revisited using the new methodology[7]. The reaction takes place when the potentially formed neutral particle after a Larsen-Borgnakke trial redistribution of the total collision energy including the reaction energy is at the electronic energy level corresponding to that just above the activation energy of the endothermic reaction.

\section{Endothermic and Exothermic Exchange Reactions Involving Charged Species}

In exchange reactions involving charged species, the predominant mechanism is the breaking of a bond of one species (either neutral or charged) in order to exchange one atom to bond with the original atom, just as with an exchange reaction involving only neutral particles. Therefore, the logic for performing this reaction involving charged species should be identical to that involving only neutral species. An endothermic exchange reaction takes place 
when the vibrational level of the colliding molecule after a trial Larsen-Borgnakke redistribution of energy resulting from the sum of the relative translational energy and the vibrational energy of the molecule is one level above the level corresponding to the activation energy $E_{a}$

$$
i_{a}=\operatorname{int}\left[E_{a} / k_{B} \Theta_{v}\right]
$$

where $i_{a}$ is the first vibrational level above the energy threshold of the reaction, $E_{a}$ is the activation energy, $k_{B}$ is Boltzmann's constant and $\Theta_{v}$ is the characteristic vibrational temperature.

Again, since the exothermic exchange reaction occurs with atoms/molecules of similar mass, the detailed balance model[5] is acceptable for use, but should be revisited using the new methodology[7]. An exothermic exchange reaction therefore takes place when the potentially formed molecule after a Larsen-Borgnakke trial redistribution of the total collision energy including the reaction energy is at the vibrational state corresponding to the activation energy as in Equation (4).

\section{APPLICATION TO PARTICULAR REACTIONS}

The new chemistry models as discussed above are investigated by examining their ability to reproduce rates of chemical reactions for which measurements exist. The DSMC models are applied in a two-dimensional code where the results are accumulated over all cells to approximate a zero-dimensional simulation. The test gas is comprised of eleven species: $\mathrm{O}, \mathrm{N}, \mathrm{O}_{2}, \mathrm{~N}_{2}, \mathrm{NO}, \mathrm{O}^{+}, \mathrm{N}^{+}, \mathrm{O}_{2}{ }^{+}, \mathrm{N}_{2}{ }^{+}, \mathrm{NO}^{+}$, and $e$. The simulations were run at varying temperatures between $10,000 \mathrm{~K}$ and $60,000 \mathrm{~K}$ at a number density of $1 \mathrm{e} 23 / \mathrm{m}^{3}$ in an adiabatic box $0.002 \mathrm{~m}$ on a side with 300,000 particles. During the simulations, relaxation of the particles (including rotational, vibrational and electronic) was allowed to proceed as usual, but when a reaction was determined to take place, the number of reactions was advanced by one but the simulators were left unchanged, so no energy was added to or taken away from the flow. In the figures included in this section, the lines are values from quoted rates in the literature while the symbols are the values that were sampled from DSMC. For a reaction $A+B \rightarrow C+D$, the forward reaction rate is calculated as

$$
k_{f}=\frac{\frac{d n_{A}}{d t}}{n_{A} n_{B}}
$$

where the numerator is the change in number density of species $A$ due to the reaction in the sampled time and the denominator is the product of the number densities of species $A$ and $B$.

The capability of these techniques to reproduce measured equilibrium reaction rates will now be investigated. However, the uncertainty of the currently available measured rates for atmospheric hypersonic flows can be large. Most of the experimental data on which reaction-rate sets are based were obtained for low temperatures (2000$7000 \mathrm{~K}$ ). Through careful study and comparison with numerical simulations, Park[4] suggests that the experimentally obtained reaction rates can be extrapolated up to $30,000 \mathrm{~K}$.

The uncertainty of these measured rates is not always reported and in some cases is unknown. The problem becomes even more severe in the extrapolated portion of the temperature range ( $\mathrm{T} \geq 10,000 \mathrm{~K})$. Park[4] reports that some rates measured for the same reaction differ by more than one order of magnitude, which will be apparent in the figures that follow.

\section{Associative Ionization}

Due to their low threshold energies, associative ionization reactions play an important role in determining the level of ionization and structure of the flow field at hypersonic reentry conditions. There exists very little experimental or computational data in the literature regarding the associative ionization reactions in air. The associative ionization reaction of $N+O$ has the lowest energy threshold, however the associative ionization of $N+N$ and $O+O$ are also very important mechanisms in the current application. The sampled DSMC reaction rates of these reactions are compared to measured rates from the literature[4, 9-11] in Figs. 1, 2, and 3, respectively. The discrete points represent the sampled DSMC rates,

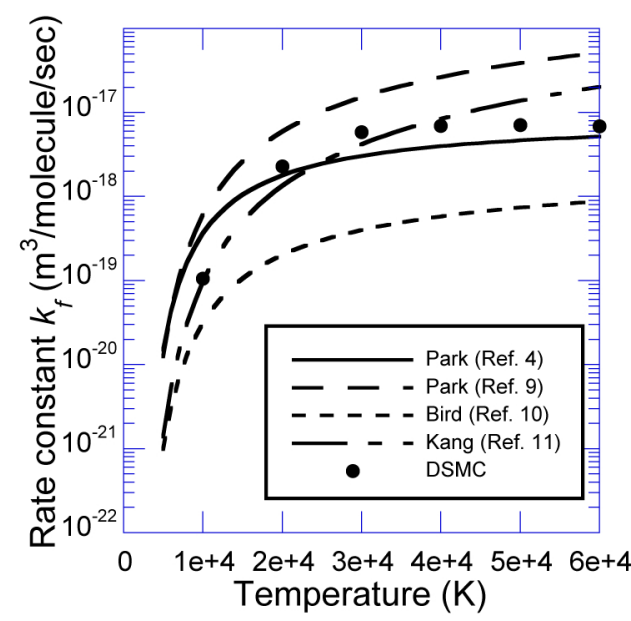

FIGURE 1. Associative ionization rate of atomic nitrogen and atomic oxygen. 


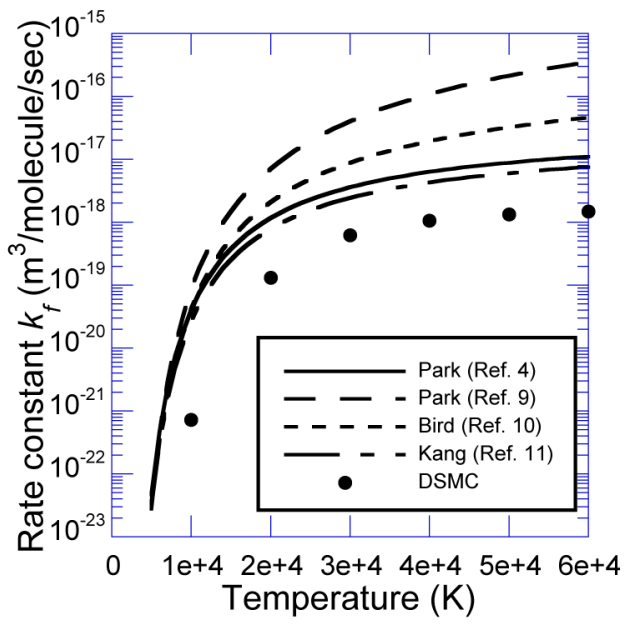

FIGURE 2. Associative ionization rate of atomic nitrogen.

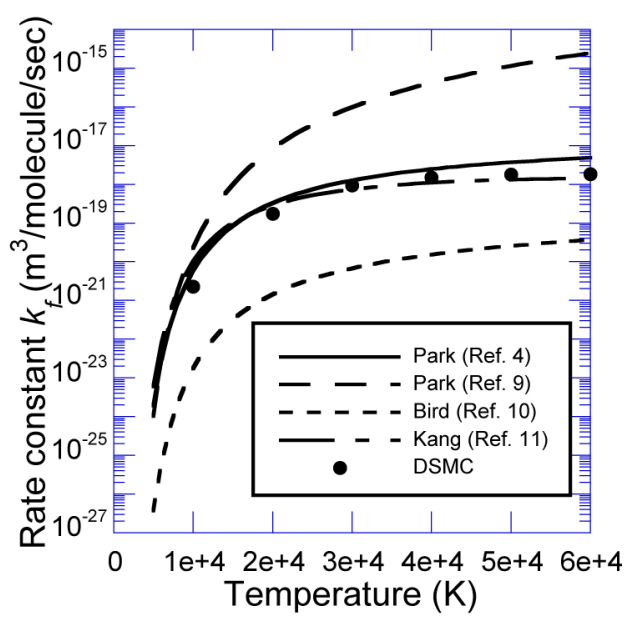

FIGURE 3. Associative ionization rate of atomic oxygen.

while the lines represent the experimental measurements corresponding to the reference listed in the figure legend. As discussed earlier, there is a wide spread in the reaction rates reported in the literature. Even so, the sampled reaction rates for $N+O$ and $O+O$ (Figs. 1 and 3) go through the middle of the experimental scatter, while the sampled rates for $N+N$, while lower than the experimental data, are still within an order of magnitude of the lowest measured rate. Given the assumed measurement uncertainty, the DSMC values are in good agreement with measured rates.

\section{Ionization}

Another important reaction mechanism for ionized, hypersonic reentry problems is the ionization of an atom or molecule by direct impact. There is very limited data in the literature for heavy particle impact ionization (such as $N$ $+N \rightarrow N^{+}+N+e$ ), so these reactions won't be discussed in this work. There is, however, an appreciable amount of data related to electron impact ionization reactions, especially for atomic species. The electron impact ionization reaction rates of $N$ and $N_{2}$ are presented in Figs. 4 and 5, respectively, compared to values from the literature[4, 10, 12-16]. As with the associative ionization data, there is a wide spread (almost three orders of magnitude) in the available reaction rate curves for the ionization of $N$, but the sampled DSMC data goes through the middle of the scatter. There weren't nearly as many references for the electron impact ionization of $N_{2}$, but the comparison shown in Fig. 5 is still mostly within one order of magnitude (especially in the temperature range of interest for rarefied,

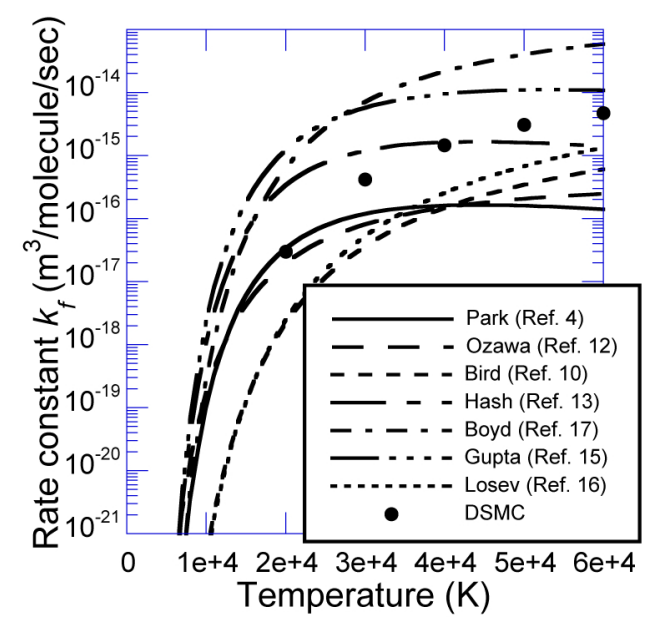

FIGURE 4. Electron impact ionization rate of atomic nitrogen.



FIGURE 5. Electron impact ionization rate of molecular nitrogen. 




FIGURE 6. Endothermic charge exchange reaction $N_{2}+$ $N^{+} \rightarrow N_{2}^{+}+N$.



FIGURE 7. Exothermic charge exchange reaction $\mathrm{N}_{2}^{+}+$ $N \rightarrow N_{2}+N^{+}$.

ionized, hypersonic reentry problems). Once again, the sampled DSMC values are in good agreement with the measured rates.

\section{Endothermic and Exothermic Charge Exchange}

In charge exchange reactions, a singly charged ion (such as $N^{+}$or $O^{+}$) collides with a neutral atom or molecule and captures one of its electrons, thereby becoming a neutral atom. Only a small amount of energy is transferred to the electron donor, so the newly created neutral retains most of the original energy of the ion. These can therefore be an important mechanism for the creation of high energy neutral particles. The measured reaction rates from the endothermic and exothermic charge exchange reactions corresponding to $N_{2}+N^{+} \rightarrow N_{2}{ }^{+}+N$ are shown in Figs. 6 and 7, respectively, compared to values from the literature[10,11, 16, 17]. Again, the spread in the reported Arrhenius curves is quite large, but the sampled DSMC reaction rates are in good comparison with the reported values.

\section{Endothermic and Exothermic Exchange Reactions Involving Charged Species}

The remainder of relevant chemical reactions involve various exchange reactions involving charged species. As an example, the sampled endothermic and exothermic reaction rates related to $\mathrm{O}+\mathrm{NO}^{+} \rightarrow \mathrm{O}_{2}^{+}+\mathrm{N}$ are presented in Figs. 8 and 9 , respectively, compared to values from the literature[18]. The references for these types of reactions

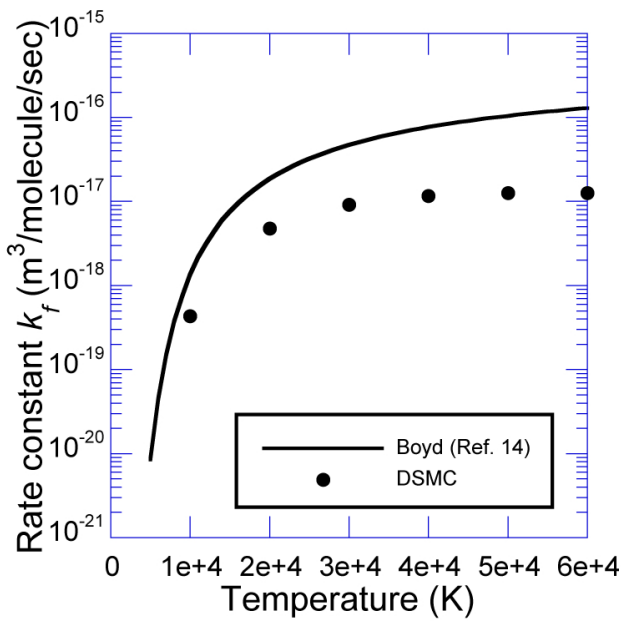

FIGURE 8. Endothermic charge exchange reaction $O+$ $\mathrm{NO}^{+} \rightarrow \mathrm{O}_{2}^{+}+\mathrm{N}$.

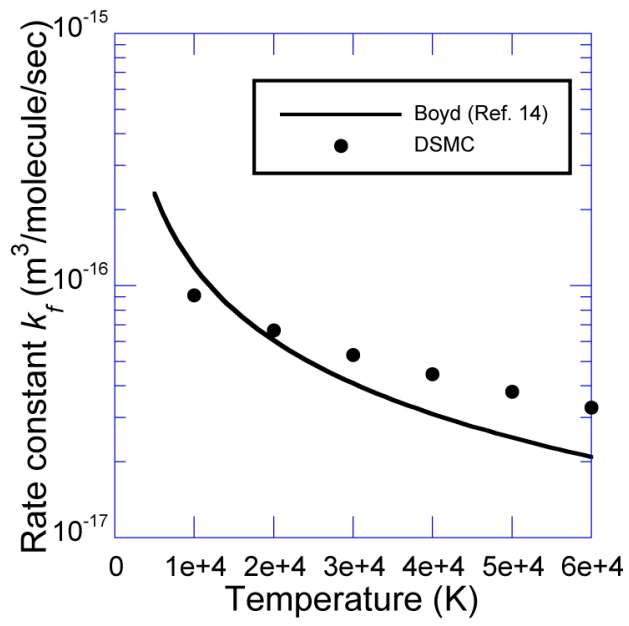

FIGURE 9. Exothermic charge exchange reaction $\mathrm{O}_{2}{ }^{+}+$ $\mathrm{N} \rightarrow \mathrm{O}+\mathrm{NO}^{+}$. 
are scarce, but for both the endothermic and exothermic reactions shown, the comparison between the sampled DSMC rate and the reported curve are within an order of magnitude.

\section{CONCLUDING REMARKS}

Extensions to the recently introduced kinetic-theory approach for computing chemical-reaction rates have been proposed for reactions including charged species. These reactions include ionization, associative ionization, endothermic charge exchange, and other exchange reactions involving charged species. These new models do not use measured macroscopic reaction rates to calibrate adjustable parameters. Instead, they make use of the principles of microscopic reversibility and molecular-level energy exchange to predict the probability that a chemical reaction occurs during a collision between to particles.

For several reaction types, the DSMC models produce equilibrium reaction rates that are in good qualitative and quantitative agreement with the best available measured or extrapolated reaction rates. The uncertainty of the experiments do not allow an absolute assessment of the DSMC models through these comparisons. However, the differences between the most reliable reaction rates and the corresponding DSMC values are usually less than an order of magnitude, which is generally within the uncertainty of the measurements and often certainly within the scatter of equilibrium rates quoted in the literature. The advantage of the new kinetic-theory approach for computing reaction rates becomes apparent if the rate data is not available or if the application requires the extrapolation of the data to temperatures well outside the range of the measurements.

\section{REFERENCES}

1. Carlson, A.B. and H.A. Hassan, Radiation Modeling with Direct Simulation Monte Carlo. Journal of Thermophysics and Heat Transfer, 1992. 6: p. 631-636.

2. Berghausen, A.K., J.C. Taylor, and H.A. Hassan, Direct Simulation of Shock Front Radiation in Air. Journal of Thermophysics and Heat Transfer, 1996. 10(3): p. 6.

3. Kossi, K.K. and I.D. Boyd, Detailed computation of Ultraviolet Spectra in Rarefied Hypersonic Flow. Journal of Thermophysics and Heat Transfer, 1998. 35: p. 653-659.

4. Park, C., Nonequilibrium Hypersonic Aerothermodynamics. 1990, New York: Wiley.

5. Bird, G.A. A Comparison of Collision Energy-based and Temperature-based Procedures in DSMC. in Rarefied Gas Dynamics, 26th Symposium. 2009. Kyoto, Japan: American Institue of Physics.

6. Gallis, M.A., R.B. Bond, and J.R. Torczynski, A kinetic-theory approach for computing chemical-reaction rates in upperatmosphere hypersonic flows. The Journal of Chemical Physics, 2009. 131(12): p. 124311.

7. Bird, G.A. Chemical Reactions in DSMC. in Rarefied Gas Dynamics, 27th Symposium. 2010. Pacific Grove, CA: American Institute of Physics.

8. Liechty, D.S. and M.J. Lewis, Treatment of Electronic Energy Level Transition and Ionization Following the Particle-Based Chemistry Model. 2010, AIAA 2010-449.

9. Park, C., Chemical-Kinetic Problems of Future NASA Missions. 1991, AIAA 1991-464.

10. Bird, G.A., Nonequilibrium Radiation During Re-Entry at 10 km/s. 1987, AIAA 1987-1543.

11. Kang, S.W. and M.G. Dunn, Theoretical and Measured Electron-Density Distributions for the RAM Vehicle at High Altitudes. 1972, AIAA 1972-689.

12. Ozawa, T., J. Zhong, and D.A. Levin, Development of kinetic-based energy exchange models for noncontinuum, ionized hypersonic flows. Physics of Fluids, 2008. 20.

13. Hash, D., et al., FIRE II Calculations for Hypersonic Nonequilibrium Aerothermodynamics Code Verification: DPLR, LAURA and US3D. 2007, AIAA 2007-605.

14. Gupta, R.N., Navier-Stokes and Viscous Shock-Layer Solutions for Radiating Hypersonic Flows. 1987, AIAA $1987-1576$.

15. Losev, S.A., et al., Thermochemical Nonequilibrium Kinetic Models in Strong Shock Waves on Air. 1994, AIAA $1994-1990$.

16. Boyd, I.D. and T. Gokcen, Computation of Axisymmetric and Ionized Flows Using Particle and Continuum Methods. 1993 , AIAA 1993-729.

17. Phelps, A.V., Cross Sections and Swarm Coefficients for Nitrogen Ions and Neutrals in N2 and Argon Ions and Neutrals in Ar for Energies from $0.1 \mathrm{eV}$ to $10 \mathrm{keV}$. J. Phys. Chem. Ref. Data, 1991. 20: p. 557.

18. Boyd, I.D., Modeling of Plasma Formation in Rarefied Hypersonic Entry Flows. 2007, AIAA 2007-206. 